\title{
Methicillin-resistant Staphylococcus lugdunensis carrying SCCmec type V misidentified as MRSA
}

\begin{abstract}
Staphylococcus lugdunensis is a rare cause of severe infections and clinical manifestations are similar to those related to $S$. aureus infection. We describe a hospital-acquired bacteremia due to methicillin-resistant Staphylococcus lugdunensis, misidentified as methicillin-resistant S. aureus. The oxacillin MIC was $16 \mu \mathrm{g} / \mathrm{mL}$ and the mecA gene and SCCmec type V were determined by PCR. Although treatment had been appropriated, the patient died after rapid progressive respiratory failure and another nosocomial sepsis. It is important not only to identify S. lugdunensis in view of its clinical course, but also to determine its susceptibility to oxacillin by detecting the mecA gene or its product.
\end{abstract}

Keywords: Staphylococcus; methicillin resistance; bacteremia.

[Braz J Infect Dis 2011;15(3):293-295] @Elsevier Editora Ltda.

\section{CASE REPORT}

A 73-year old female with systemic arterial hypertension and chronic obstructive pulmonary disease (COPD) GOLD IV was admitted to the Emergency Department of the University Hospital 'Clementino Fraga Filho' (HUCFF), in March 2005. HUCFF, a tertiary-care teaching hospital with 490 beds, is affiliated to the Federal University of Rio de Janeiro, Rio de Janeiro City, Brazil. The patient had asthenia, cough, fever, purulent secretions and worsening of dyspnea, but the chest $\mathrm{x}$-ray showed no pulmonary infiltrates. Diagnosis of acute exacerbation and upper respiratory infection were made. Bronchodilators, glucocorticoids, gatifloxacin and non-invasive ventilation were initiated. Respiration did not recover, and mechanical ventilation was needed. After completing the course of antimicrobial therapy the patient recovered. However, two cultures from blood collected at day 7 of therapy detected Gram-positive bacteria. Bacterial contamination of blood was initially suspected since the patient had improved and precise identification of the sites of blood collection was not possible. Initially, methicillin-resistant S. aureus (MRSA) and Streptococcus alpha hemolytic were identified in the hospital laboratory three days after blood collection and the strains were sent to our laboratory for confirmation. After the initial clinical improvement, the patient presented fever, leucocytosis and hypotension. The central line catheter was removed, and vancomycin and amikacin were included in the treatment, according to the local microbiology pattern and the previous isolation. The nosocomial sepsis was probably related to the central line catheter, ${ }^{1}$ although abdominal imaging were not performed. The transthoracic echocardiogram result was normal. Another set of blood cultures drawn on the day when sepsis was diagnosed detected Escherichia coli and S. epidermidis. The patient had fast and progressive respiratory failure and despite intense medical treatment she expired on day 18 of hospitalization.

The isolate obtained from the first blood culture was characterized as MRSA in the hospital laboratory by VITEK (BioMerieux). Gram staining, catalase test and latex agglutination clumping factor and DNAse detection were performed as complementary tests. Antimicrobial susceptibility testing (VITEK, BioMerieux) revealed resistance to oxacillin, penicillin and chloramphenicol.
Authors

Eliezer Menezes Pereira ${ }^{1}$ Ricardo Pinto Schuenck ${ }^{2}$ Simone Aranha Nouér Kátia Regina Netto dos Santos ${ }^{4}$

${ }^{1} \mathrm{MD}$, Professor, Prof. Denise Bello Applied Microbiology and Fermentation Processes Laboratory, Instituto Federal de Educação Ciência e Tecnologia do Rio de Janeiro, RJ, Brazil ${ }^{2} \mathrm{MD}$, Professor, Department of Pathology, Universidade Federal do Espírito Santo, Vitória, ES ${ }^{3} \mathrm{MD}$, Professor, Medical School, Hospital Universitário Clementino Fraga Filho, Universidade Federal do Rio de Janeiro (UFRJ), RJ, Brazil

${ }^{4} \mathrm{MD}$, Professor, Instituto de Microbiologia Prof. Paulo de Góes, UFRJ, RJ, Brazil

Submitted on: 12/09/2010 Approved on: 12/12/2010

Correspondence to: Kátia Regina Netto dos Santos Av. Carlos Chagas Filho, 373

Ilha do Fundão, Rio de Janeiro, RJ - 21941-902 santoskrn@micro.ufrj.br

Financial Support: CNPq, FAPERJ, CAPES, PRONEX, FUJB

We declare no conflict of interest. 
In our laboratory, the isolate presented slightly yellow colonies and showed beta-hemolysis on sheep blood agar. It gave a positive reaction for clumping factor, but the tube coagulase test was negative. Then, the isolate was evaluated using the method based on the conventional reference tests ${ }^{2}$ and the ornithine decarboxylase production, an enzyme produced only by $S$. lugdunensis strains. ${ }^{3}$ To confirm the species identification, the $f b l$ gene, which encodes a fibrinogen binding protein of $S$. lugdunensis was amplified by PCR as previously published. ${ }^{2}$ A 425 bp amplicon was obtained after PCR amplification of template DNA extracted from reference strain S. lugdunensis DSMZ 4804 and the clinical isolate. Antimicrobial susceptibility was evaluated according to CLSI guidelines. The isolate was resistant to oxacillin, cefoxitin, penicillin and chloramphenicol by disk diffusion test. Oxacillin MIC, determined by the E-test method, was $16 \mu \mathrm{g} / \mathrm{mL}$. Initially, the mecA gene was detected by PCR, and the SCCmec typing was performed by multiplex PCR of the ccr type and mec class, using the method described by Kondo et al. ${ }^{4}$ The isolate was ccr type 5 and $m e c$ class C, characterizing the SCCmec type V. The $804 \mathrm{bp}$ amplicon (corresponding to ccr5 gene) and 518 bp (corresponding to class $\mathrm{C}$ mec gene) generated in PCR reaction were confirmed by sequencing and comparison with GenBank database.

This is the first case report of a methicillin-resistant $S$. lugdunensis (MRSL) isolate carrying the SCCmec type V causing a systemic infection. To our knowledge, this is the fourth report in the literature describing $S$. lugdunensis carrying the mecA gene. Kawaguchi et al. ${ }^{5}$ detected the mecA gene in one of two strains of $S$. lugdunensis isolated from blood during a laboratory surveillance in Japan, but no description of clinical infection was provided by them. In 2003, Tee et al..$^{6}$ reported a case of MRSL causing bloodstream infection in a neonate. In 2008, Tan, $\mathrm{Ng}$ and $\mathrm{He}^{7}$ found five (4.7\%) S. lugdunensis strains carrying the mecA gene in a collection of 106 clinical isolates. In Brazil, this is the first report of a MRSL isolate.

S. lugdunensis was firstly described by Freney ${ }^{8}$ and is associated with several infections, mainly skin/soft tissues infections and endocarditis. ${ }^{9}$ In the clinical setting it frequently causes illness that has been described as acute, aggressive and destructive - more in keeping with coagulase-positive staphylococci than characteristically indolent coagulase-negative staphylococcal infections, especially endocarditis, where the majority of infections involve native or prosthetic valves, being the mortality rates high. ${ }^{10}$ Therefore, its aggressive nature resembles $S$. aureus. S. lugdunensis should be suspected when a staphylococcal isolate is positive in the clumping factor test but negative in the tube coagulase test. ${ }^{6}$ The isolation rates of S. lugdunensis are about $10 \%$ for non-S. epidermidis coagulase negative isolates. ${ }^{3}$ However, in a recent study, Böcher et al. ${ }^{11}$ showed that the misidentification of $S$. lugdunensis could be contributing to this low rate. They improved the identification of S. lugdunensis using the Columbia sheep-horse agar inoculation as an additional test. To reinforce this fact we evaluated the bacterial diagnostic provided for 5,360 blood cultures performed in our hospital, between 2000 and 2009 (data not shown). No S. lugdunensis isolate was detected, suggesting that this pathogen is being misidentified.

In the present case report we observed a clear misidentification of a MRSL isolate as community MRSA. Both species present similar colony morphology and blood-agar hemolysis. Interestingly, in addition to being positive by the clumping factor agglutination test, S. lugdunensis strains are also positive for DNAse, like S. aureus strains. This shows the importance to employ tube coagulase testing for staphylococcal characterization. For confirmation the ornithine-decarboxylase test is also important, since S. lugdunensis is the only staphylococcus positive in this assay. The susceptibility pattern of the isolate indicated a community-associated pathogen. Only resistance for $\beta$-lactams and chloramphenicol were observed. Moreover, this non-multiresistance has been related to the SCCmec type $\mathrm{V}$, often found in community isolates. This case report confirms the isolation of non-multiresistant $S$. lugdunensis carrying the SCCmec V as a cause of a serious hospital-acquired infection which was misdiagnosed as non-multiresistant MRSA in a setting with nosocomial transmission of the latter.

\section{REFERENCES}

1. Mermel LA, Allon M, Bouza E et al. Clinical practice guidelines for the diagnosis and management of intravascular catheter-related infection: 2009 Update by the Infectious Diseases Society of America. Clin Infect Dis. 2009; 49:1-45.

2. Pereira EM, Oliveira FL, Schuenck RP, Zoletti GO, dos Santos KR. Detection of Staphylococcus lugdunensis by a new species-specific PCR based on the $f b l$ gene. FEMS Immunol Med Microbiol. 2010; 58:295-29.

3. Herchline TE, Ayers LW. Occurrence of Staphylococcus lugdunensis in consecutive clinical cultures and relationship of isolation to infection. J Clin Microbiol. 1991; 29:419-21.

4. Kondo Y, Ito T, Ma XX et al. Combination of multiplex PCRs for staphylococcal cassette chromosome mec type assignment: rapid identification system for $m e c$, ccr, and major differences in junkyard regions. Antimicrob Agents Chemother. 2007; 51;264-74.

5. Kawaguchi E, Minamide W, Mori H, Igmi H. The taxonomic distribution, characteristic and susceptibility against antimicrobial agents of methicillin-resistant staphylococci isolated from blood. Kansenshogaku Zasshi 1996; 70:1147-53.

6. Tee WS, Soh SY, Lin R, Loo L. Staphylococcus lugdunensis carrying the mecA gene causes catheter-associated bloodstream infection in premature neonate. J Clin Microbiol. 2003; 41:519-20.

7. Tan TY, Ng SY, He J. Microbiological characteristics, presumptive identification, and antibiotic susceptibilities of Staphylococcus lugdunensis. J Clin Microbiol. 2008; 46:2393-5. 
8. Freney J, Brun Y, Bes M et al. Staphylococcus lugdunensis and Staphylococcus schleiferi sp. nov, two species from human clinical specimens. Int J Syst Bacteriol. 1988; 38:168-72.

9. Frank KL, Del Pozo JL, Patel R. From clinical microbiology to infection pathogenesis: how daring to be different works for Staphylococcus lugdunensis. Clin Microbiol Rev. 2008; 21:111-133.
10. Ebright JR, Penugonda N, Brown W. Clinical experience with Staphylococcus lugdunensis bacteremia: a retrospective analysis. Diagn Microbiol Infec Dis. 2004; 48:17-21.

11. Böcher S, Tønning B, Skov RL, Prag J. Staphylococcus lugdunensis, a common cause of skin and soft tissue infections in the community. J Clin Microbiol. 2009;47:946-50. 